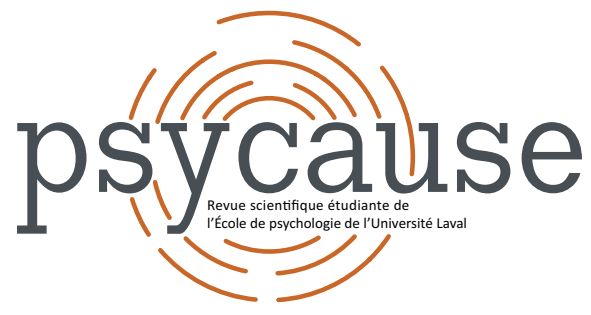

\title{
PSYCAUSE
}

Revue scientifique étudiante de l'École de psychologie de l'Université Laval

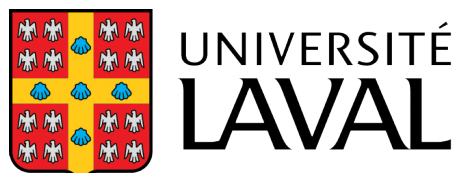

Faculté des sciences sociales École de psychologie

\section{LA DÉTECTION DES CONTINGENCES CHEZ LES ENFANTS ÂGÉS ENTRE SIX ET 18 MOIS}

\author{
Laura FORTIN ${ }^{1,2, \star}$, Catherine ST-PIERRE ${ }^{1,2}$, Élodie ST-PIERRE ${ }^{1,2}$, Émilie LANGLOIS 1,2 \\ et George M. TARABULSY ${ }^{1,2}$ \\ ${ }^{1}$ École de Psychologie, Université Laval \\ ${ }^{2}$ Centre de recherche universitaire sur les jeunes et les familles (CRUJeF) \\ *laura.fortin.4@ulaval.ca
}

\section{Pour citer l'article}

Fortin, L., St-Pierre, C., St-Pierre, É., Langlois, É., \& Tarabulsy, G. M. (2019). La détection des contingences chez les enfants âgés entre six et 18 mois. Psycause: Revue scientifique étudiante de l'École de psychologie de l'Université Laval, 9(2), 13-15. 
Belleville, G., Guay, S., \& Marchand, A. (2011). Persistence of sleep disturbances following cognitive-behavior therapy for posttraumatic stress disorder. Journal of Psychosomatic Research, 70(4), 318-327. doi:10.1016/j. jpsychores.2010.09.022

Bradley, R., Greene, J., Russ, E., Dura, L., \& Westen, D. (2005). Multi-dimensional meta-analysis of psychotherapy for refugees. American Journal of Psychiatry, 162, 214-227. doi:10.1176/appi.ajp.162.2.214

Casement, M. D., \& Swanson, L. M. (2012). A meta-analysis of imagery rehearsal for post-trauma nightmares: Effects on nightmare frequency, sleep quality, and posttraumatic stress. Clinical Psychology Review, 32(6), 566-574. doi:10.1016/j.cpr.2012.06.009
Cusack, K., Jonas, D. E., Forneris, C. A., Wines, C., Sonis, J., Middleton, J. C., ... Gaynes, B. N. (2016). Psychological treatments for adults with posttraumatic stress disorder: A systematic review and meta-analysis. Clinical Psychology Review, 43(290), 128-141. doi:10.1016/j.cpr.2015.10.003

Forbes, D., Phelps, A. J., McHugh, A. F., Debenham, P., Hopwood, M., \& Creamer, M. (2003). Imagery rehearsal in the treatment of posttraumatic nightmares in Australian veterans with chronic combat-related PTSD: 12-month follow-up data. Journal of Traumatic Stress, 16(5), 509-513. doi:10.1023/A:1025718830026

Hembree, E. A., \& Foa, E. B. (2000). Posttraumatic stress disorder: Psychological factors and psychosocial interventions. The Journal of Clinical Psychiatry, 61(7), 33-39.

\title{
Pour citer l'article
}

Fiset, N., Flores-Tremblay, T., Gaboury, M.-P., \& Belleville, G. (2019). L'efficacité à long terme de la thérapie cognitive-comportementale du trouble de stress post-traumatique avec ou sans traitement des cauchemars. Psycause: Revue scientifique étudiante de l'École de psychologie de I'Université Laval, 9(2), 11-13.

\section{LA DÉTECTION DES CONTINGENCES CHEZ LES ENFANTS ÂGÉS ENTRE SIX ET 18 MOIS}

\author{
Laura FORTIN ${ }^{1,2, *}$, Catherine ST-PIERREE ${ }^{1,2}$, Élodie ST-PIERREE ${ }^{1,2}$, Émilie LANGLOIS ${ }^{1,2}$ et George M. TARABULSY, \\ ${ }^{1}$ École de Psychologie, Université Laval; ${ }^{2}$ Centre de recherche universitaire sur les jeunes et les familles (CRUJeF) \\ *laura.fortin.4@ulaval.ca
}

\section{Mots-clés : Détection des contingences; Développement de l'enfant; Nourrissons; Risque psychosocial}

La détection des contingences (DC) se définit comme la détection de la relation temporelle entre l'apparition de deux événements ou entre un comportement et un événement (Northrup, 2017). Ce concept, élémentaire dans la vie quotidienne, permet d'anticiper des événements et d'organiser ses comportements en fonction de prédictions. Diverses caractéristiques ont une influence sur les résultats à une tâche de DC (Lemelin, Tarabulsy \& Provost, 2002; Northrup, 2017), et le niveau de risque psychosocial pourrait faire partie de ces aspects. Un milieu à risque se caractérise par un ensemble de facteurs susceptibles de diminuer la prévisibilité de l'environnement de l'enfant et d'augmenter la probabilité qu'il soit exposé à des éléments considérés négatifs pour son développement (Neuhauser, 2018). Les conséquences liées à l'exposition au risque psychosocial ont des répercussions sur le développement émotionnel, social et cognitif de l'enfant; des domaines pour lesquels la DC est importante (Spieker, Larson, Lewis, White \& Gilchrist, 1997). Cependant, peu d'études ont exploré le lien entre l'écologie du développement de l'enfant et la DC, et aucune n'a utilisé de devis longitudinal. L'objectif de la présente étude est d'examiner comment la DC évolue dans le temps et comment elle peut être influencée par le risque psychosocial auquel l'enfant est exposé.

\section{Méthode}

La présente recherche s'inscrit dans le cadre d'une étude longitudinale de plus grande envergure menée au Québec par Tarabulsy et Provost entre 1999 et 2001. De cet échantillon, 130 dyades mère-enfant, comprenant 78 mères adolescentes définies comme étant à haut risque sur le plan psychosocial et 52 mères adultes définies comme étant à faible risque, prennent part aux tâches de DC en laboratoire. Les critères d'inclusion chez l'enfant sont un poids à la naissance supérieur à $2500 \mathrm{~g}$ et une absence d'anomalies physiques ou congénitales.

La tâche de DC est composée de deux phases. La phase du niveau de base est d'une minute et il n'y a pas de renforcement. La phase de contingence est de trois minutes, et chaque mouvement de bras est renforcé, pendant quatre 
secondes, par l'apparition de visages d'enfants souriants sur un écran et le déclenchement d'un segment d'une chanson populaire pour enfant. Lorsqu'un mouvement de bras se produit pendant la période de quatre secondes, cette réponse est enregistrée et une nouvelle période de quatre secondes commence. Lors de la Tâche de détection des contingences avant un an, un ruban attaché au poignet de l'enfant enregistre les mouvements, alors que dans la Tâche de détection des contingences à 15 et 18 mois, l'enfant effectue une compression sur un bouton. La Figure 1 illustre ces deux tâches.

\section{Tâche de détection des contingences avant 1 an}

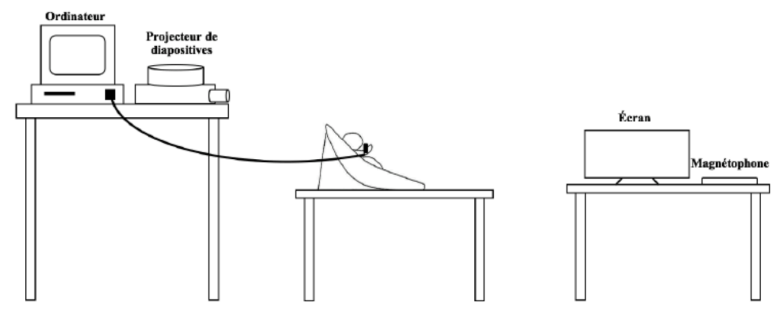

Tâche de détection des contingences à 15 et 18 mois
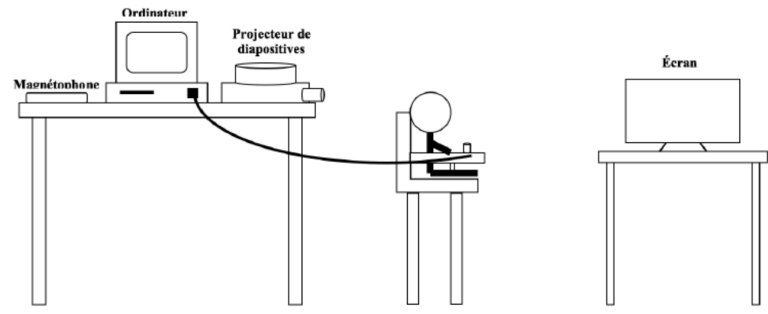

Figure 1. Illustration de la Tâche de détection des contingences avant 1 an et de la Tâche de détection des contingences à 15 et 18 mois.

\section{Résultats et discussion}

Les enfants âgés de six mois ayant été exclus des analyses en raison d'un problème technique, seule la période entre 10 et 18 mois est prise en compte.

Les résultats démontrent d'abord qu'il n'existe pas de relation significative entre les taux d'apprentissage des contingences à 10 et 15 mois $(r=0,047), 10$ et 18 mois $(r=-0,094)$, et 15 et 18 mois $(r=-0,129)$. Ce résultat suggère une absence de stabilité dans les taux de DC, qui pourrait s'expliquer par l'influence de caractéristiques instables, telles que l'humeur de l'enfant au moment de la tâche ou le niveau d'éveil (Sullivan \& Lewis, 2003). Il est également possible que la DC joue un rôle différent au cours du développement et, qu'à cet âge, des stratégies du traitement de l'information plus sophistiquées soient préférablement employées pour repérer les liens dans l'environnement.

Par la suite, les différences individuelles dans la capacité de $D C$ entre 10 et 18 mois sont illustrées à la Figure 2 par deux patrons de trajectoires d'apprentissage, soit constante pour $90,7 \%$ des enfants et linéaire croissante pour $9,3 \%$ des enfants. La trajectoire constante serait tributaire d'un effet de moyenne et non d'une stabilité du taux d'apprentissage de la DC, les trajectoires individuelles variant de façon aléatoire. La trajectoire linéaire croissante suggère quant à elle que certains enfants auraient un comportement distinct lors de la phase du niveau de base, qui simulerait des taux d'apprentissage supérieurs, sans pour autant refléter une meilleure capacité de DC (Millar \& Weir, 2015).

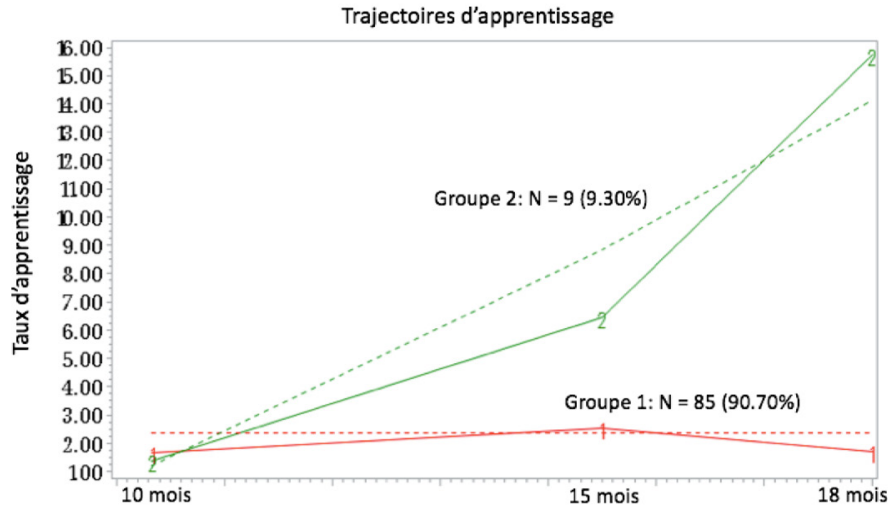

Figure 2. Illustration des trajectoires des taux d'apprentissage chez les enfants âgés de 10 à 18 mois.

Finalement, les résultats montrent qu'il n'existe pas de relation significative entre le taux d'enfants appartenant à chacune des trajectoires d'apprentissage et leur niveau de risque psychosocial. Un tel résultat est compatible avec les données de Kaplan, Bachorowski, Smoski \& Hudenko (2002), où les enfants de mères dépressives obtenaient des résultats similaires aux enfants de mères non dépressives à une tâche de DC en situation de stimulation contingente. II est possible qu'au cours du développement, des différences pourraient se manifester entre les deux groupes d'enfants au sein d'autres aspects du traitement de l'information liés à la DC. De futures études sont nécessaires afin d'identifier de tels mécanismes, et pourraient permettre de mieux circonscrire le rôle de la DC au cours du développement, tout en affinant notre compréhension de son lien avec le risque psychosocial.

\section{Références}

Kaplan, P. S., Bachorowski, J.-A., Smoski, M. J., \& Hudenko, W. J. (2002). Infants of Depressed Mothers, Although Competent Learners, Fail to Learn in Response to Their Own Mothers' Infant-Directed Speech. Psychological Science, 13(3), 268-271.

Lemelin, J.-P., Tarabulsy, G. M., \& Provost, M. A. (2002). Relations between measures of irritability and contingency detection at 6 months. Infancy, 3(4), 543-554. 
Millar, W. S., \& Weir, C. (2015). Baseline response levels are a nuisance in infant contingency learning. Infant and Child Development, 24(5), 506-521.

Neuhauser, A. (2018). Predictors of maternal sensitivity in at-risk families. Early Child Development and Care, 188(2), 126142.

Northrup, J. B. (2017). Contingency detection in a complex world: A developmental model and implications for atypical development. International Journal of Behavioral Deve- lopment, 41(6), 723-734.

Spieker, S. J., Larson, N. C., Lewis, S. M., White, R. D., \& Gilchrist, L. (1997). Children of adolescent mothers: Cognitive and behavioral status at age six. Child and Adolescent Social Work Journal, 14(5), 335-364.

Sullivan, M. W., \& Lewis, M. (2003). Contextual determinants of anger and other negative expressions in young infants. Developmental psychology, 39(4), 693.

\title{
Pour citer l'article
}

Fortin, L., St-Pierre, C., St-Pierre, É., Langlois, É., \& Tarabulsy, G. M. (2019). La détection des contingences chez les enfants âgés entre six et 18 mois. Psycause: Revue scientifique étudiante de l'École de psychologie de l'Université Laval, 9(2), 13-15.

\section{LA RELATION ENTRE LES PENSÉES POSITIVES ET NÉGATIVES, LES STRATÉGIES DE RÉGULATION ÉMOTIONNELLE ET LA PEUR DE RÉCIDIVE DU CANCER}

\author{
Samuele DALLAIRE-HABEL ${ }^{1, *}$, Geneviève OUELLET' ${ }^{1}$, Frédéric THÉRIAULT-COUTURE ${ }^{1}$, Véronique MASSICOTTE ${ }^{1,2,3}$, \\ Hans IVERS ${ }^{1,2,3}$, Anne-Josée GUIMOND ${ }^{1,2,3}$ et Josée SAVARD ${ }^{1,2,3}$ \\ ${ }^{1}$ École de Psychologie, Université Laval; ${ }^{2}$ Centre de recherche du CHU de Québec-Université Laval; ${ }^{3}$ Centre de recherche sur \\ le cancer de l'Université Laval \\ *samuele.dallaire-habel.1@ulaval.ca
}

\section{Mots-clés : Cancer; Peur de la récidive du cancer; Pensées; Régulation émotionnelle; Modèle cognitif}

La peur de la récidive du cancer (PRC), qui se définit comme la peur, l'inquiétude ou une préoccupation quant à la possibilité que le cancer revienne ou progresse, est fréquemment rapportée par les patientes atteintes d'un cancer du sein (Lebel et coll., 2016). Certains travaux ont examiné le rôle des pensées dans la PRC et montrent qu'une plus grande tendance à entretenir des pensées négatives et à anticiper négativement le futur (pessimisme) est positivement associée à la PRC (p. ex., Deimling, Bowman, Sterns, Wagner \& Kahana, 2006). II a été proposé dans la littérature dédiée au grand public que la pensée positive pourrait s'avérer une option de rechange avantageuse. Toutefois, une étude sur l'optimisme irréaliste, un construit apparenté, suggère qu'elle pourrait être associée à de la détresse psychologique (Hurt, Weinman, Lee \& Brown, 2012). Par ailleurs, le modèle des processus de régulation émotionnelle (RE) de Gross et Thompson (2007) souligne la pertinence d'examiner le rôle médiateur des stratégies de RE dans la relation entre les pensées et la PRC en proposant que l'interprétation cognitive d'une situation amène l'individu à adopter certaines stratégies de RE afin d'exercer une influence sur ses émotions. La présente étude, menée chez des femmes atteintes d'un cancer du sein, a pour objectifs: (a) d'examiner les associations transversales et prospectives entre les pensées négatives et positives et la PRC; et (b) d'explorer le rôle médiateur des stratégies de $R E$, soit la réévaluation cognitive, la suppression émotionnelle et l'évitement expérientiel, dans la relation entre le type de pensées (négatives et positives) et la PRC.

\section{Méthode}

L'échantillon est composé de 81 femmes recrutées à l'HôtelDieu de Québec (Centre hospitalier universitaire de QuébecUniversité Laval), ayant reçu un diagnostic de cancer du sein. Les participantes sont âgées en moyenne de 59 ans (É-T $=8,5$ ) et la majorité est traitée pour un cancer de stade 1 $(66,3 \%)$. Les patientes complètent l'Inventaire de peur de récidive du cancer, le Questionnaire des pensées et anticipations au sujet du cancer (PAC), le Questionnaire de régulation émotionnelle et le Questionnaire d'acceptation et d'action-Il à la maison avant le début des traitements de radiothérapie (T1) et après leur fin ( $T 2$; six semaines après le T1). Puisque le PAC mesure les pensées et anticipations quant au traitement à recevoir, il est administré au T1 uniquement. 